\title{
Alternative proofs of some formulas for two tridiagonal determinants
}

\author{
Feng Qi \\ Institute of Mathematics, \\ Henan Polytechnic University, China \\ College of Mathematics, Inner Mongolia \\ University for Nationalities, China \\ Department of Mathematics, \\ College of Science, Tianjin Polytechnic \\ University, China \\ email: qifeng618@gmail.com, \\ qifeng618@hotmail.com, \\ qifeng618@qq. com \\ https://qifeng618.wordpress.com
}

\author{
Ai-Qi Liu \\ Department of Mathematics, \\ Sanmenxia Polytechnic, China \\ email: smxptliu@hotmail.com
}

\begin{abstract}
In the paper, the authors provide five alternative proofs of two formulas for a tridiagonal determinant, supply a detailed proof of the inverse of the corresponding tridiagonal matrix, and provide a proof for a formula of another tridiagonal determinant. This is a companion of the paper [F. Qi, V. Čerňanová, and Y. S. Semenov, Some tridiagonal determinants related to central Delannoy numbers, the Chebyshev polynomials, and the Fibonacci polynomials, Politehn. Univ. Bucharest Sci. Bull. Ser. A Appl. Math. Phys. 81 (2019), in press.
\end{abstract}

2010 Mathematics Subject Classification: Primary 47B36; Secondary 11B39, 11B83, 11C08, 11C20, 11Y55, 15B36, 26C99

Key words and phrases: alternative proof, tridiagonal determinant, tridiagonal matrix, inverse 


\section{Introduction}

For $c \in \mathbb{C}$ and $k \in \mathbb{N}$, define the $k \times k$ tridiagonal matrix $M_{k}(c)$ by

$$
M_{k}(c)=\left(\begin{array}{cccccccc}
c & 1 & 0 & 0 & \cdots & 0 & 0 & 0 \\
1 & c & 1 & 0 & \cdots & 0 & 0 & 0 \\
0 & 1 & c & 1 & \cdots & 0 & 0 & 0 \\
\vdots & \vdots & \vdots & \vdots & \ddots & \vdots & \vdots & \vdots \\
0 & 0 & 0 & 0 & \cdots & c & 1 & 0 \\
0 & 0 & 0 & 0 & \cdots & 1 & c & 1 \\
0 & 0 & 0 & 0 & \cdots & 0 & 1 & c
\end{array}\right)_{k \times k}
$$

and denote the determinant $\left|M_{k}(c)\right|$ of the $k \times k$ tridiagonal matrix $M_{k}(c)$ by $\mathrm{D}_{\mathrm{k}}(\mathrm{c})$. In [7, Remark 4.4], the explicit expression

$$
D_{k}(-6)=\frac{1}{6^{k}} \sum_{\ell=0}^{k}(-1)^{\ell} 6^{2 \ell}\left(\begin{array}{c}
\ell \\
k-\ell
\end{array}\right)
$$

was derived from some results in [7, Theorem 1.2] for the Cauchy products of central Delannoy numbers, where $\left(\begin{array}{l}p \\ q\end{array}\right)=0$ for $q>p \geq 0$. For information on central Delannoy numbers, please refer to the papers $[6,7]$ and plenty of references cited therein. In [7, Remar 4.4], the authors guessed that the explicit formula

$$
D_{k}(c)=(-1)^{k} \sum_{\ell=0}^{k}(-1)^{\ell} c^{2 \ell-k}\left(\begin{array}{c}
\ell \\
k-\ell
\end{array}\right)=\sum_{m=0}^{k}(-1)^{m} c^{k-2 m}\left(\begin{array}{c}
k-m \\
m
\end{array}\right)
$$

should be valid for all $c \in \mathbb{C}$ and $k \in \mathbb{N}$ and claimed that the equality (1) can be verified by induction on $k \in \mathbb{N}$ straightforwardly.

In the paper [6], the authors discovered a generating function of the sequence $D_{k}(c)$, provided an analytic proof of the explicit formula (1), established a simple formula for computing the tridiagonal determinant $D_{k}(c)$, found a determinantal expression for $D_{k}(c)$, presented the inverse of the symmetric tridiagonal matrix $M_{k}(c)$, connected $D_{k}(c)$ with the Chebyshev polynomials $[6,9,11]$ and the Fibonacci numbers and polynomials $[1,6,8]$, reviewed computation of general diagonal determinants, supplied two new formulas for computing general diagonal determinants, generalized central Delannoy numbers $[6,7]$, and represented the Cauchy product of the generalized central Delannoy numbers [6] in terms of $D_{k}(c)$.

In this paper, we pay our attention on the following four conclusions. 
Theorem 1 ([6, Theorem 2.2]) For $\mathrm{k} \geq 0$ and $\mathrm{c} \in \mathbb{C}$, the formula (1) is valid.

Theorem 2 ([6, Theorem 3.1]) For $c \in \mathbb{C}, \alpha=\frac{1}{\beta}=\frac{\mathrm{c}+\sqrt{\mathrm{c}^{2}-4}}{2}$, and $\mathrm{k} \geq 0$, the tridiagonal determinant $\mathrm{D}_{\mathrm{k}}(\mathrm{c})$ can be computed by

$$
D_{k}(c)= \begin{cases}\frac{\alpha^{k+1}-\beta^{k+1}}{\alpha-\beta}, & c \neq \pm 2 \\ k+1, & c=2 \\ (-1)^{k}(k+1), & c=-2\end{cases}
$$

Theorem 3 ([6, Theorem 5.1]) For $k \in \mathbb{N}$, the inverse of the symmetric tridiagonal matrix $\mathrm{M}_{\mathrm{k}}(\mathrm{c})$ can be computed by $\mathrm{M}_{\mathrm{k}}^{-1}(\mathrm{c})=\left(\mathrm{R}_{\mathrm{ij}}\right)_{\mathrm{k} \times \mathrm{k}}$, where

$$
R_{i j}= \begin{cases}-\frac{\left(\lambda^{i}-\mu^{i}\right)\left(\lambda^{k-j+1}-\mu^{k-j+1}\right)}{(\lambda-\mu)\left(\lambda^{k+1}-\mu^{k+1}\right)}, & c \neq \pm 2 \\ (-1)^{i+j} \frac{i(k-j+1)}{k+1}, & c=2 \\ -\frac{i(k-j+1)}{k+1}, & c=-2\end{cases}
$$

for $i<j, R_{i j}=R_{j i}$ for $i>j$, and $\lambda$ and $\mu$ are defined by

$$
\lambda=\frac{1}{\mu}=\frac{2}{\sqrt{c^{2}-4}-c}=-\alpha=-\frac{1}{\beta} .
$$

Theorem $4([6$, Section 8]) For $n \in \mathbb{N}$ and $\mathrm{a}, \mathrm{b}, \mathrm{c} \in \mathbb{C}$, we have

$$
\begin{aligned}
D_{n} & =\left|\begin{array}{cccccc}
a & b & 0 & \cdots & 0 & 0 \\
c & a & b & \cdots & 0 & 0 \\
0 & c & a & \cdots & 0 & 0 \\
\vdots & \vdots & \vdots & \ddots & \vdots & \vdots \\
0 & 0 & 0 & \cdots & a & b \\
0 & 0 & 0 & \cdots & c & a
\end{array}\right|_{n \times n} \\
& = \begin{cases}\frac{\left(a+\sqrt{a^{2}-4 b c}\right)^{n+1}-\left(a-\sqrt{a^{2}-4 b c}\right)^{n+1}}{(n+1)\left(\frac{a}{2}\right)^{n},}, & a^{2} \neq 4 b c ; \\
2^{n+1} \sqrt{a^{2}-4 b c} & a^{2}=4 b c .\end{cases}
\end{aligned}
$$


In Section 2 of this paper, we will supply two alternative proofs of Theorem 1. In Section 3, we will provide three alternative proofs of Theorem 2. In Section 4, we will present a detailed proof of Theorem 3. In Section 5, we will provide a proof of Theorem 4. In the last section of this paper, we will list several remarks.

\section{Two alternative proofs of Theorem 1}

Now we are in a position to supply two alternative proofs of Theorem 1.

Proof. [First alternative proof of Theorem 1] Let $\mathrm{D}_{0}(\mathrm{c})=1$. Theorem 2.1 in [6] states that the sequence $D_{k}(c)$ for $k \geq 0$ can be generated by

$$
F_{c}(t)=\frac{1}{t^{2}-c t+1}=\sum_{k=0}^{\infty} D_{k}(c) t^{k}
$$

By the formula for the sum of a geometric progression, the generating function $F_{c}(t)$ can be expanded as

$$
\mathrm{F}_{\mathrm{c}}(\mathrm{t})=\sum_{\ell=0}^{\infty}(-1)^{\ell}\left(\mathrm{t}^{2}-\mathrm{ct}\right)^{\ell}=\sum_{\ell=0}^{\infty} \sum_{\mathrm{m}=0}^{\ell}(-1)^{\mathrm{m}}\left(\begin{array}{c}
\ell \\
\mathrm{m}
\end{array}\right) \mathrm{c}^{\ell-\mathrm{m} \mathrm{t}^{\ell+m}}
$$

for $\left|t^{2}-c t\right|<1$. Hence, it follows for $k \geq 0$ that

$$
\begin{aligned}
{\left[\mathrm{F}_{\mathrm{c}}(\mathrm{t})\right]^{(\mathrm{k})} } & =\sum_{\ell=0}^{\infty} \sum_{\mathrm{m}=0}^{\ell}(-1)^{\mathrm{m}}\left(\begin{array}{c}
\ell \\
\mathrm{m}
\end{array}\right) \mathrm{c}^{\ell-\mathrm{m}}\left(\mathrm{t}^{\ell+\mathrm{m}}\right)^{(\mathrm{k})} \\
& \rightarrow \sum_{\ell=0}^{\infty} \sum_{\mathrm{m}=0}^{\ell}(-1)^{\mathrm{m}}\left(\begin{array}{c}
\ell \\
\mathrm{m}
\end{array}\right) \mathrm{c}^{\ell-\mathrm{m}} \lim _{\mathrm{t} \rightarrow 0}\left(\mathrm{t}^{\ell+\mathrm{m}}\right)^{(\mathrm{k})} \\
& =(-1)^{\mathrm{k}} \mathrm{k} ! \sum_{\ell=0}^{k}(-1)^{\ell}\left(\begin{array}{c}
\ell \\
k-\ell
\end{array}\right) c^{2 \ell-k}
\end{aligned}
$$

for $\left|t^{2}-c t\right|<1$ and as $t \rightarrow 0$. The formula (1) is thus proved.

Proof. [Second alternative proof of Theorem 1] Taking $k=\ell+m$ in (5) leads to

$$
F_{c}(t)=\sum_{k=0}^{\infty}\left[\sum_{\ell=0}^{k}(-1)^{k-\ell}\left(\begin{array}{c}
\ell \\
k-\ell
\end{array}\right) c^{2 \ell-k}\right] t^{k}=\sum_{k=0}^{\infty} D_{k}(c) t^{k}
$$

for $\left|t^{2}-c t\right|<1$. The formula (1) is proved again. The proof of Theorem 1 is complete. 


\section{Three alternative proofs of Theorem 2}

We now start out to provide three alternative proofs of Theorem 2.

Proof. [First alternative proof of Theorem 2] It is clear that the generating function $F_{c}(t)$ in $(4)$ can be rewritten as $F_{c}(t)=\frac{1}{t-\alpha} \frac{1}{t-\beta}$. By virtue of the Leibniz theorem for the product of two functions, we have

$$
\begin{aligned}
& {\left[F_{c}(t)\right]^{(k)}=\left(\frac{1}{t-\alpha} \frac{1}{t-\beta}\right)^{(k)}=\sum_{\ell=0}^{k}\left(\begin{array}{l}
k \\
\ell
\end{array}\right)\left(\frac{1}{t-\alpha}\right)^{(\ell)}\left(\frac{1}{t-\beta}\right)^{(k-\ell)} } \\
= & \sum_{\ell=0}^{k}\left(\begin{array}{l}
k \\
\ell
\end{array}\right) \frac{(-1)^{\ell} \ell !}{(t-\alpha)^{\ell+1}} \frac{(-1)^{k-\ell}(k-\ell) !}{(t-\beta)^{k-\ell+1}} \rightarrow \sum_{\ell=0}^{k}\left(\begin{array}{l}
k \\
\ell
\end{array}\right) \frac{(-1)^{\ell} \ell !}{(-\alpha)^{\ell+1}} \frac{(-1)^{k-\ell}(k-\ell) !}{(-\beta)^{k-\ell+1}} \\
= & k ! \sum_{\ell=0}^{k} \frac{1}{\alpha^{\ell+1}} \frac{1}{\beta^{k-\ell+1}}=\frac{k !}{\beta^{k}} \sum_{\ell=0}^{k}\left(\frac{\beta}{\alpha}\right)^{\ell}=\frac{k !}{\beta^{k}} \frac{1-(\beta / \alpha)^{k+1}}{1-\beta / \alpha}=k ! \frac{\alpha^{k+1}-\beta^{k+1}}{\alpha-\beta}
\end{aligned}
$$

as $t \rightarrow 0$. The formula (2) is thus proved.

Proof. [Second alternative proof of Theorem 2] The generating function $F_{c}(t)$ can also be rewritten as

$$
F_{c}(t)=\frac{1}{\alpha-\beta}\left(\frac{1}{t-\alpha}-\frac{1}{t-\beta}\right) .
$$

Then a straightforward computation reveals

$$
\begin{aligned}
& {\left[F_{c}(t)\right]^{(k)}=\frac{1}{\alpha-\beta}\left[\frac{(-1)^{k} k !}{(t-\alpha)^{k+1}}-\frac{(-1)^{k} k !}{(t-\beta)^{k+1}}\right]} \\
& \rightarrow-k ! \frac{1}{\alpha-\beta}\left(\frac{1}{\alpha^{k+1}}-\frac{1}{\beta^{k+1}}\right)=k ! \frac{\alpha^{k+1}-\beta^{k+1}}{\alpha-\beta}
\end{aligned}
$$

as $t \rightarrow 0$. The proof of Theorem 2 is complete.

Proof. [Third alternative proof of Theorem 2] The formula for the sum of a geometric progression yields

$$
\frac{1}{t-\alpha}=-\sum_{k=0}^{\infty} \frac{t^{k}}{\alpha^{k+1}} \quad \text { and } \quad \frac{1}{t-\beta}=-\sum_{k=0}^{\infty} \frac{t^{k}}{\beta^{k+1}}
$$

for $|t|<\min \{|\alpha|,|\beta|\}$. Thus, in view of $\alpha \beta=1$ and (6), we obtain

$$
F_{c}(t)=\frac{1}{\alpha-\beta} \sum_{k=0}^{\infty}\left(\frac{1}{\beta^{k+1}}-\frac{1}{\alpha^{k+1}}\right) t^{k}=\sum_{k=0}^{\infty} \frac{\alpha^{k+1}-\beta^{k+1}}{\alpha-\beta} t^{k}=\sum_{k=0}^{\infty} D_{k}(c) t^{k}
$$

for $|t|<\min \{|\alpha|,|\beta|\}$. The formula (2) is thus proved. The proof of Theorem 2 is complete. 


\section{A detailed proof of Theorem 3}

We now present a detailed proof of Theorem 3.

In the paper [2], the inverse of the symmetric tridiagonal matrix $M_{k}(c)$ was discussed. We denote the inverse matrix of $M_{k}(c)$ by $M_{k}^{-1}(c)=\left(R_{i j}\right)_{k \times k}$. Then, basing on discussions in [2, Eq. (9)], one can see without difficulty that the elements $R_{i j}$ can be represented as

$$
R_{i j}=(-1)^{i+j} \frac{D_{i-1}(c) D_{k-j}(c)}{D_{k}(c)}, \quad 1 \leq i<j \leq k
$$

and $R_{i j}=R_{j i}$ for $1 \leq j<i \leq k$. Making use of the formula (2) yields

$$
\begin{aligned}
R_{i j} & = \begin{cases}(-1)^{i+j} \frac{\alpha^{i-1+1}-\beta^{i-1+1}}{\alpha-\beta} \frac{\alpha^{k-j+1}-\beta^{k-j+1}}{\alpha-\beta}, & c \neq \pm 2 \\
(-1)^{i+j} \frac{( \pm 1)^{i-1}\left(i-1+\beta^{k+1}\right.}{\alpha-\beta} & ( \pm 1)^{k}(k+1)\end{cases} \\
& = \begin{cases}(-1)^{i+j} \frac{\left(\alpha^{i}-\beta^{i}\right)\left(\alpha^{k-j+1}-\beta^{k-j+1}\right)}{(\alpha-\beta)\left(\alpha^{k+1}-\beta^{k+1}\right)}, & c \neq \pm 2 \\
(-1)^{i+j}( \pm 1)^{i-j-1} \frac{i(k-j+1)}{k+1}, & c= \pm 2\end{cases} \\
& = \begin{cases}-\frac{\left[(-\alpha)^{i}-(-\beta)^{i}\right]\left[(-\alpha)^{k-j+1}-(-\beta)^{k-j+1}\right]}{[(-\alpha)-(-\beta)]\left[(-\alpha)^{k+1}-(-\beta)^{k+1}\right]}, & c \neq \pm 2 \\
(-1)^{i+j} \frac{i(k-j+1)}{k+1}, & c=2 \\
-\frac{i(k-j+1)}{k+1}, & c=-2\end{cases} \\
& = \begin{cases}(-1)^{i+j} \frac{\left(\lambda^{i}-\mu^{i}\right)\left(\lambda^{k-j+1}-\mu^{k-j+1}\right)}{k+1}, & c \neq \pm 2 \\
-\frac{i(k-j+1)}{k+1}, & c=2\end{cases}
\end{aligned}
$$

for $1 \leq i<j \leq k$. The proof of Theorem 3 is complete. 


\section{A proof of Theorem 4}

The determinant $D_{n}$ satisfies the recurrence relation $D_{n}=a D_{n-1}-b c D_{n-2}$. Solving the equation $x^{2}-a x+b c=0$ reaches to two roots $\alpha=\frac{a+\sqrt{a^{2}-4 b c}}{2}$ and $\beta=\frac{a-\sqrt{a^{2}-4 b c}}{2}$. These two roots satisfy $\alpha+\beta=a$ and $\alpha \beta=b c$. Then by the above recurrence relation one can write

$$
\begin{aligned}
D_{n}-\alpha D_{n-1}=\beta\left[D_{n-1}\right. & \left.-\alpha D_{n-2}\right]=\beta^{2}\left[D_{n-2}-\alpha D_{n-3}\right]=\cdots \\
= & \beta^{n-2}\left[D_{2}-\alpha D_{1}\right]=\beta^{n-2}\left[\left(a^{2}-b c\right)-\alpha a\right]=\beta^{n} .
\end{aligned}
$$

Similarly, one can deduce that $D_{n}-\beta D_{n-1}=\alpha^{n}$. Accordingly, when $\alpha \neq \beta$, that is, $a^{2} \neq 4 b c$, one finds $(\alpha-\beta) D_{n}=\alpha^{n+1}-\beta^{n+1}$, that is,

$$
D_{n}=\frac{\alpha^{n+1}-\beta^{n+1}}{\alpha-\beta}=\frac{\left(a+\sqrt{a^{2}-4 b c}\right)^{n+1}-\left(a-\sqrt{a^{2}-4 b c}\right)^{n+1}}{2^{n+1} \sqrt{a^{2}-4 b c}} .
$$

When $\alpha=\beta$, that is, $a^{2}=4 b c$, we have

$$
\begin{gathered}
D_{n}=\alpha^{n}+\alpha D_{n-1}=\alpha^{n}+\alpha\left(\alpha^{n-1}+\alpha D_{n-2}\right)=\cdots=(n-1) \alpha^{n}+\alpha^{n-1} D_{1} \\
=(n-1) \alpha^{n}+\alpha^{n-1}(2 \alpha)=(n+1) \alpha^{n}=(n+1)\left(\frac{a}{2}\right)^{n} .
\end{gathered}
$$

The formula (3) is thus proved. The proof of Theorem 4 is complete.

\section{Several remarks}

Finally, we list several remarks on tridiagonal determinants.

Remark 1 The identities

$$
\mathcal{D}_{k}(c) \triangleq\left|\begin{array}{ccccccc}
-c & 1 & 0 & \cdots & 0 & 0 & 0 \\
2 & -2 c & 1 & \cdots & 0 & 0 & 0 \\
0 & 6 & -3 c & \cdots & 0 & 0 & 0 \\
\vdots & \vdots & \vdots & \ddots & \vdots & \vdots & \vdots \\
0 & 0 & 0 & \cdots & -(k-2) c & 1 & 0 \\
0 & 0 & 0 & \cdots & (k-1)(k-2) & -(k-1) c & 1 \\
0 & 0 & 0 & \cdots & 0 & k(k-1) & -k c
\end{array}\right|
$$




$$
\begin{aligned}
& =(-1)^{k} k !\left|\begin{array}{cccccccc}
c & 1 & 0 & 0 & \cdots & 0 & 0 & 0 \\
1 & c & 1 & 0 & \cdots & 0 & 0 & 0 \\
0 & 1 & c & 1 & \cdots & 0 & 0 & 0 \\
\vdots & \vdots & \vdots & \vdots & \ddots & \vdots & \vdots & \vdots \\
0 & 0 & 0 & 0 & \cdots & c & 1 & 0 \\
0 & 0 & 0 & 0 & \cdots & 1 & c & 1 \\
0 & 0 & 0 & 0 & \cdots & 0 & 1 & c
\end{array}\right|_{k \times k} \\
& =\frac{k !}{c^{k}} \sum_{\ell=0}^{k}(-1)^{\ell} c^{2 \ell}\left(\begin{array}{c}
\ell \\
k-\ell
\end{array}\right)= \begin{cases}k ! \frac{\lambda^{k+1}-\mu^{k+1}}{\lambda-\mu}, & c \neq \pm 2 \\
(-1)^{k}(k+1) !, & c=2 \\
(k+1) !, & c=-2\end{cases}
\end{aligned}
$$

are neither trivial nor obvious, where $\lambda=\frac{1}{\mu}=\frac{2}{\sqrt{c^{2}-4}-\mathrm{c}}=-\alpha=-\frac{1}{\beta}$. The determinant $\mathcal{D}_{k}(\mathrm{c})$ satisfies

$$
\mathcal{D}_{0}(\mathrm{c})=1, \quad \mathcal{D}_{1}(\mathrm{c})=-\mathrm{c}, \quad \mathcal{D}_{2}(\mathrm{c})=2\left(\mathrm{c}^{2}-1\right),
$$

and

$$
\mathcal{D}_{\mathrm{k}}(\mathrm{c})=-\mathrm{kc} \mathcal{D}_{\mathrm{k}-1}(\mathrm{c})-\mathrm{k}(\mathrm{k}-1) \mathcal{D}_{\mathrm{k}-2}(\mathrm{c}), \quad \mathrm{k} \geq 2 .
$$

Then, if letting $\mathcal{F}_{\mathrm{c}}(\mathrm{t})=\sum_{\mathrm{k}=0}^{\infty} \mathcal{D}_{\mathrm{k}}(\mathrm{c}) \mathrm{t}^{\mathrm{k}}$, we have

$$
\begin{aligned}
\sum_{k=2}^{\infty} \mathcal{D}_{k}(c) t^{k} & =-c t \sum_{k=2}^{\infty} k \mathcal{D}_{k-1}(c) t^{k-1}-t^{2} \sum_{k=2}^{\infty} k(k-1) \mathcal{D}_{k-2}(c) t^{k-2} \\
\sum_{k=0}^{\infty} \mathcal{D}_{k}(c) t^{k} & -\mathcal{D}_{0}(c)-\mathcal{D}_{1}(c) t=-c t \sum_{k=1}^{\infty}(k+1) \mathcal{D}_{k}(c) t^{k} \\
& -t^{2} \sum_{k=0}^{\infty}(k+2)(k+1) \mathcal{D}_{k}(c) t^{k}
\end{aligned}
$$

$$
\begin{aligned}
\mathcal{F}_{c}(t)-1+c t= & -c t \frac{d}{d t}\left[\sum_{k=1}^{\infty} \mathcal{D}_{k}(c) t^{k+1}\right]-t^{2} \frac{d^{2}}{d t^{2}}\left[\sum_{k=0}^{\infty} \mathcal{D}_{k}(c) t^{k+2}\right], \\
\mathcal{F}_{c}(t)-1+c t= & -c t \frac{d}{d t}\left[t \sum_{k=1}^{\infty} \mathcal{D}_{k}(c) t^{k}\right]-t^{2} \frac{d^{2}}{d t^{2}}\left[t^{2} \sum_{k=0}^{\infty} \mathcal{D}_{k}(c) t^{k}\right], \\
\mathcal{F}_{c}(t)-1+c t=- & c t \frac{d}{d t}\left[t\left(\mathcal{F}_{c}(t)-1\right)\right]-t^{2} \frac{d^{2}}{d t^{2}}\left[t^{2} \mathcal{F}_{c}(t)\right], \\
& t^{4} \mathcal{F}_{c}^{\prime \prime}(t)+t^{2}(4 t+c) \mathcal{F}_{c}^{\prime}(t)+\left(2 t^{2}+c t+1\right) \mathcal{F}_{c}(t)-1=0 .
\end{aligned}
$$


This means that the generating function of the sequence $\mathcal{D}_{\mathrm{k}}(\mathrm{c})=(-1)^{\mathrm{k}} \mathrm{k} ! \mathrm{D}_{\mathrm{k}}(\mathrm{c})$ is the solution of the second order linear ordinary differential equation

$$
t^{4} f^{\prime \prime}(t)+t^{2}(4 t+c) f^{\prime}(t)+\left(2 t^{2}+c t+1\right) f(t)-1=0
$$

with initial values $\mathrm{f}(0)=1$ and $\mathrm{f}^{\prime}(0)=-\mathrm{c}$. This differential equation is solvable, but its solution is not elementary.

Remark 2 The method used in the proof of [6, Theorem 3.1] can not be applied to the sequence $\mathcal{D}_{k}(\mathrm{c})$, since its recurrence relation (7) is not a homogeneous linear recurrence relation with constant coefficients.

Remark 3 The central Delannoy numbers $\mathrm{D}(\mathrm{k})$ were generalized in [10] as

$$
D_{a, b}(k)=\frac{1}{\pi} \int_{a}^{b} \frac{1}{\sqrt{(t-a)(b-t)}} \frac{1}{t^{k+1}} d t, \quad k \geq 0, \quad b>a>0
$$

and, by [7, Lemma 2.4], we find that $\mathrm{D}_{\mathrm{a}, \mathrm{b}}(\mathrm{k})$ can be generated by

$$
\frac{1}{\sqrt{(x+a)(x+b)}}=\sum_{k=0}^{\infty} D_{a, b}(k) x^{k} .
$$

By virtue of conclusions in [4, Section 2.4] and [3, Remark 4.1], the generalized central Delannoy numbers $\mathrm{D}_{\mathrm{a}, \mathrm{b}}(\mathrm{k})$ for $\mathrm{k} \geq 0$ can be computed by

$$
D_{a, b}(k)=\frac{1}{a^{k+1}}{ }_{2} F_{1}\left(k+1, \frac{1}{2} ; 1 ; 1-\frac{b}{a}\right), \quad 2 a>b>a>0, \quad k \geq 0,
$$

where ${ }_{2} \mathrm{~F}_{1}$ is the classical hypergeometric function which is a special case of the generalized hypergeometric series

$$
{ }_{p} F_{q}\left(a_{1}, \ldots, a_{p} ; b_{1}, \ldots, b_{q} ; z\right)=\sum_{n=0}^{\infty} \frac{\left(a_{1}\right)_{n} \ldots\left(a_{p}\right)_{n}}{\left(b_{1}\right)_{n} \ldots\left(b_{q}\right)_{n}} \frac{z^{n}}{n !}
$$

for complex numbers $a_{i} \in \mathbb{C}$ and $b_{i} \in \mathbb{C} \backslash\{0,-1,-2, \ldots\}$, for positive integers $\mathrm{p}, \mathrm{q} \in \mathbb{N}$, and for

$$
(x)_{\ell}= \begin{cases}\prod_{k=0}^{\ell-1}(x+k), & \ell \geq 1 \\ 1, & \ell=0\end{cases}
$$

which is called the rising factorial of $x \in \mathbb{R}$.

Remark 4 This paper and [6] are extracted from different parts of the preprint [5]. 


\section{References}

[1] C.-P. Chen, A.-Q. Liu, and F. Qi, Proofs for the limit of ratios of consecutive terms in Fibonacci sequence, Cubo Mat. Educ. 5 (3) (2003), 23-30.

[2] G. Y. Hu and R. F. O'Connell, Analytical inversion of symmetric tridiagonal matrices, J. Phys. A 29 (7) (1996), 1511-1513; Available online at http://dx.doi.org/10.1088/0305-4470/29/7/020.

[3] F. Qi and B.-N. Guo, The reciprocal of the weighted geometric mean is a Stieltjes function, Bol. Soc. Mat. Mex. (3) 24 (1) (2018), 181-202; Available online at http://dx.doi.org/10.1007/s40590-016-0151-5.

[4] F. Qi and V. Čerňanová, Some discussions on a kind of improper integrals, Int. J. Anal. Appl. 11 (2) (2016), 101-109.

[5] F. Qi, V. Čerňanová, and Y. S. Semenov, On tridiagonal determinants and the Cauchy product of central Delannoy numbers, ResearchGate Working Paper (2016), available online at http://dx.doi.org/10.13140/RG.2. 1.3772 .6967$.

[6] F. Qi, V. Čerňanová, and Y. S. Semenov, Some tridiagonal determinants related to central Delannoy numbers, the Chebyshev polynomials, and the Fibonacci polynomials, Politehn. Univ. Bucharest Sci. Bull. Ser. A Appl. Math. Phys. 81 (2019), in press.

[7] F. Qi, V. Čerňanová, X.-T. Shi, and B.-N. Guo, Some properties of central Delannoy numbers, J. Comput. Appl. Math. 328 (2018), 101-115; Available online at https://doi.org/10.1016/j.cam.2017.07.013.

[8] F. Qi and B.-N. Guo, Expressing the generalized Fibonacci polynomials in terms of a tridiagonal determinant, Matematiche (Catania) 72 (1) (2017), 167-175; Available online at https://doi.org/10.4418/2017.72.1.13.

[9] F. Qi, D.-W. Niu, and D. Lim, Notes on the Rodrigues formulas for two kinds of the Chebyshev polynomials, HAL archives (2018), available online at https://hal.archives-ouvertes.fr/hal-01705040.

[10] F. Qi, X.-T. Shi, and B.-N. Guo, Some properties of the Schröder numbers, Indian J. Pure Appl. Math. 47 (4) (2016), 717-732; Available online at http://dx.doi.org/10.1007/s13226-016-0211-6. 
[11] F. Qi, Q. Zou, and B.-N. Guo, Some identities and a matrix inverse related to the Chebyshev polynomials of the second kind and the Catalan numbers, Preprints 2017, 2017030209, 25 pages; Available online at https://doi. org/10.20944/preprints201703.0209.v2.

Received: June 19, 2018 